Hand Hygiene Noncompliance and the Cost of Hospital-Acquired Methicillin-Resistant Staphylococcus aureus Infection •

Author(s): Keith L. Cummings, MD, MBA, Deverick J. Anderson, MD, MPH, Keith S. Kaye , MD, MPH

Source: Infection Control and Hospital Epidemiology, Vol. 31, No. 4 (April 2010), pp. 357-364 Published by: The University of Chicago Press on behalf of The Society for Healthcare Epidemiology of America

Stable URL: http://www.jstor.org/stable/10.1086/651096

Accessed: 10/06/2011 13:12

Your use of the JSTOR archive indicates your acceptance of JSTOR's Terms and Conditions of Use, available at http://www.jstor.org/page/info/about/policies/terms.jsp. JSTOR's Terms and Conditions of Use provides, in part, that unless you have obtained prior permission, you may not download an entire issue of a journal or multiple copies of articles, and you may use content in the JSTOR archive only for your personal, non-commercial use.

Please contact the publisher regarding any further use of this work. Publisher contact information may be obtained at http://www.jstor.org/action/showPublisher?publisherCode=ucpress.

Each copy of any part of a JSTOR transmission must contain the same copyright notice that appears on the screen or printed page of such transmission.

JSTOR is a not-for-profit service that helps scholars, researchers, and students discover, use, and build upon a wide range of content in a trusted digital archive. We use information technology and tools to increase productivity and facilitate new forms of scholarship. For more information about JSTOR, please contact support@jstor.org. 


\title{
Hand Hygiene Noncompliance and the Cost of Hospital-Acquired Methicillin-Resistant Staphylococcus aureus Infection
}

\author{
Keith L. Cummings, MD, MBA; Deverick J. Anderson, MD, MPH; Keith S. Kaye, MD, MPH
}

BACKGROUND. Hand hygiene noncompliance is a major cause of nosocomial infection. Nosocomial infection cost data exist, but the effect of hand hygiene noncompliance is unknown.

ов встіve. To estimate methicillin-resistant Staphylococcus aureus (MRSA)-related cost of an incident of hand hygiene noncompliance by a healthcare worker during patient care.

DESIGN. Two models were created to simulate sequential patient contacts by a hand hygiene-noncompliant healthcare worker. Model 1 involved encounters with patients of unknown MRSA status. Model 2 involved an encounter with an MRSA-colonized patient followed by an encounter with a patient of unknown MRSA status. The probability of new MRSA infection for the second patient was calculated using published data. A simulation of 1 million noncompliant events was performed. Total costs of resulting infections were aggregated and amortized over all events.

setting. Duke University Medical Center, a 750-bed tertiary medical center in Durham, North Carolina.

ReSUlts. Model 1 was associated with 42 MRSA infections (infection rate, $0.0042 \%)$. Mean infection cost was $\$ 47,092$ (95\% confidence interval [CI], \$26,040-\$68,146); mean cost per noncompliant event was \$1.98 (95\% CI, \$0.91-\$3.04). Model 2 was associated with 980 MRSA infections (0.098\%). Mean infection cost was $\$ 53,598$ (95\% CI, \$50,098-\$57,097); mean cost per noncompliant event was $\$ 52.53$ (95\% CI, \$47.73-\$57.32). A 200-bed hospital incurs \$1,779,283 in annual MRSA infection-related expenses attributable to hand hygiene noncompliance. A $1.0 \%$ increase in hand hygiene compliance resulted in annual savings of $\$ 39,650$ to a 200-bed hospital.

CONCLUsions. Hand hygiene noncompliance is associated with significant attributable hospital costs. Minimal improvements in compliance lead to substantial savings.

Infect Control Hosp Epidemiol 2010; 31:357-364

Hospital-acquired infections cause more than 98,000 deaths annually in the United States ${ }^{1}$ and are associated with increased cost and duration of hospitalization. ${ }^{2}$ Each year, hospital-acquired infections occur in $7 \%-10 \%$ of hospitalized patients during their hospital stay. ${ }^{3}$

Compounding the issue of hospital-acquired infections is the increasing degree of resistance of pathogens to antimicrobial agents. The foremost such example is methicillinresistant Staphylococcus aureus (MRSA). Among intensive care units that report to the Centers for Disease Control and Prevention, there has been a nearly 3-fold increase in the proportion of $S$. aureus infections caused by MRSA, from $22 \%$ in 1995 to $63 \%$ in 2004 . This trend is worrisome, as MRSA infections result in greater morbidity and higher costs than do infections due to methicillin-susceptible $S$. aureus. For example, hospital-acquired bloodstream infections due to MRSA lead to a 3-fold increase in total direct costs compared with total direct costs associated with methicillin-sus- ceptible $S$. aureus infections. ${ }^{4}$ Thus, continuing increases in MRSA prevalence will cause aggregate costs related to hospital-acquired infection to increase greatly.

Noncompliance with hand hygiene recommendations is widely recognized as the most important modifiable cause of hospital-acquired infections. ${ }^{5-8}$ Indeed, in their 2008 Patient Safety Goals ${ }^{9}$ The Joint Commission requires that, as the primary means of preventing hospital-acquired infections, hospitals comply with World Health Organization and/or Centers for Disease Control and Prevention hand hygiene guidelines. ${ }^{2,10-12}$ Unfortunately, rates of compliance with hand hygiene recommendations are unacceptably low in most hospitals. ${ }^{9,12,13}$ Results from most studies suggest that overall hand hygiene compliance rates are below 50\%. ${ }^{3}$

Costs associated with hospital-acquired infections and MRSA have been widely published. ${ }^{4,14-16}$ Little is known, however, regarding the actual costs of individual behaviors that lead to these infections, such as noncompliance with hand

From the Department of Medicine, Duke University Medical Center, Durham, North Carolina (K.L.C., D.J.A.); and the Department of Medicine, Wayne State University, Detroit Medical Center, Detroit, Michigan (K.S.K.).

Received July 1, 2009; accepted September 18, 2009; electronically published February 25, 2010.

(C) 2010 by The Society for Healthcare Epidemiology of America. All rights reserved. 0899-823X/2010/3104-0007\$15.00. DOI: 10.1086/651096 
hygiene during patient care. Quantifying the cost of hand hygiene noncompliance will provide clinicians, administrators, and patient advocacy groups with concrete data that can be used to improve the accountability of hand hygiene noncompliance among healthcare workers. The purpose of this study was to quantify the cost of a single episode of hand hygiene noncompliance by a healthcare worker in a hospital setting relative to risk for MRSA transmission.

\section{METHODS}

\section{Model Design and Study Setting}

A stochastic mathematical model was constructed to simulate the outcome of a single episode of hand hygiene noncompliance. Data regarding hospital admissions and episodes of contact between patients and healthcare workers were collected from Duke University Medical Center, a 750bed tertiary care hospital in Durham, North Carolina. Other data, such as MRSA prevalence rates and rates of hand hygiene compliance, were extracted from previously published reports. ${ }^{17,18}$

The mathematical model was used to simulate a specific scenario in which a healthcare worker contacts 2 patients consecutively and fails to comply with hand hygiene guidelines after contact with the first patient (patient 1) and before contact with the second patient (patient 2). Using the model, we calculated the probability of MRSA transmission from patient 1 to the healthcare worker and then from the healthcare worker to patient 2. Embedded in this analysis is a calculation of transmission potential, which estimates the prob- ability that patient 1 was MRSA-positive and the probability that patient 2 was MRSA-negative. In addition, the model was used to calculate the probability of patient 2 developing an infection due to MRSA after becoming colonized. These probabilities were then used in a simulation of 1 million episodes of hand hygiene noncompliance.

Published data regarding hospital prevalence of MRSA, rates of hospital-acquired transmission of MRSA, and rates of hand hygiene compliance served as inputs to the model (see Table 1). Data regarding daily contacts between patients and healthcare workers and data regarding average length of hospital stay were obtained from quality improvement studies previously conducted at Duke University Medical Center and were also included as inputs to the model.

Simulations were performed under 2 different scenarios. The first scenario (the normal risk scenario) simulated hand hygiene noncompliance by a healthcare worker between contacts with 2 patients of unknown MRSA status (ie, both patients 1 and 2 may or may not have been colonized with MRSA). The second scenario (the high-risk scenario) involved hand hygiene noncompliance between contacts with 2 patients by a healthcare worker in which patient 1 was colonized or infected with MRSA and the MRSA status of patient 2 was unknown.

\section{Model Inputs, Calculations, and Simulations}

Inputs. On the basis of published data, the prevalence of MRSA in inpatient settings was estimated to be $4.63 \%$ (95\% confidence interval [CI], $4.53 \%-4.72 \%){ }^{4}$ Therefore, the prob-

TABLE 1. Model Inputs and Calculated Values

\begin{tabular}{|c|c|c|}
\hline Model input & Calculation method & $\begin{array}{l}\text { Calculated value } \\
\qquad(95 \% \mathrm{CI})\end{array}$ \\
\hline MRSA total hospital prevalence, $\%$ & $\ldots$ & $4.63(4.53-4.72)$ \\
\hline$P[\mathrm{pt}(+)]^{\mathrm{a}}$ & $\ldots$ & 0.0463 \\
\hline$P[\mathrm{pt}(-)]^{\mathrm{b}}$ & $\ldots$ & 0.9537 \\
\hline Incidence of hospital-acquired MRSA, \% & $\ldots$ & 1.43 \\
\hline Mean no. of daily room visits & $\ldots$ & $56.38(52.36-60.40)$ \\
\hline Frequency of direct contact per room visit, $\%$ & $\ldots$ & 57.24 \\
\hline Mean no. of days per hospitalization & $\ldots$ & 6.26 \\
\hline Frequency of hand hygiene compliance, $\%$ & $\ldots$ & 55.13 \\
\hline Rate of infection after colonization & $\ldots$ & 0.29 \\
\hline Cost of MRSA HAI, lognormal distribution, $\$$ & & $7,228-164,392$ \\
\hline $\begin{array}{l}\text { Projected mean no. of direct contacts per } \\
\text { patient-day }\end{array}$ & Daily room visits $\times$ (direct contacts per room visit) & 32.27 \\
\hline Projected mean no. of NDCs per patient-day & Direct contacts per patient-day $\times(1-$ compliance $)$ & $14.50(13.26-15.77)$ \\
\hline Projected mean no. of CEs per patient-day & NDCs per patient-day $\times P[p t 1(+) \cap \operatorname{pt} 2(-)]$ & $0.64(0.57-0.71)$ \\
\hline Projected mean no. of CEs per hospitalization & $($ CEs per day $) \times($ days per hospitalization $)$ & 4.01 \\
\hline $\begin{array}{l}\text { Projected mean no. of hospital-acquired MRSA } \\
\text { colonizations per CE }\end{array}$ & $\begin{array}{l}\text { Incidence of hospital-acquired MRSA } \\
\text { colonization / CEs per hospitalization }\end{array}$ & 0.0036 \\
\hline Projected mean no. of MRSA infections per CE & $\begin{array}{l}\text { (MRSA colonizations per CE }) \times \text { rate of infection } \\
\text { after colonization }\end{array}$ & 0.0010 \\
\hline
\end{tabular}

Note. CE, contaminated encounter; CI, confidence interval; HAI, hospital-acquired infection; MRSA, methicillin-resistant Staphylococcus aureus; NDC, noncompliant direct contact.

${ }^{a}$ Probability that a randomly selected patient is MRSA-positive.

b Probability that a randomly selected patient is MRSA-negative. 
ability of a random patient being MRSA-positive, $P[\mathrm{pt}(+)]$, was 0.0463 . Conversely, the probability of a random patient being MRSA-negative, $P[\mathrm{pt}(-)]$, was 0.9537 . In addition, it was estimated on the basis of published data that 31\% (95\% CI, 30\%-32\%) of MRSA cases would be detected more than 48 hours after admission and would thus be categorized as hospital-acquired. ${ }^{4}$ The transmission of MRSA in the hospital to previously uncolonized inpatients was therefore calculated to occur in $1.43 \%$ of inpatients $(0.04626 \times 0.31=0.0143)$.

Direct contact was defined as physical contact between a healthcare worker and a patient. The numbers of direct contacts per patient-day were estimated from Duke University Medical Center data and published data. ${ }^{5}$ At Duke University Medical Center in February 2008, the mean number of times a patient room was visited by a healthcare worker was 56.38 (95\% CI, 52.36-60.40) per patient-day. A recent study from another institution revealed that $57.24 \%$ of room visits involve direct patient contact. ${ }^{5}$ From these estimates, we calculated a rate of 32.27 direct contacts per patient-day. The mean days per hospitalization were calculated from Duke University Hospital data as 6.26 days per hospitalization.

The aggregate rate of hand hygiene compliance after patient room visits was estimated to be $45.1 \%$. The rate of compliance after room visits involving direct patient contact was estimated to be $55.13 \% .^{5}$ The probability of infection among newly colonized patients was estimated at $29 \% .{ }^{6}$

Published estimates of total hospital cost associated with hospital-acquired MRSA infection span a broad range: mean infection cost estimates are $\$ 9,275-\$ 110,493$, and median infection cost estimates are $\$ 5,885-\$ 49,734 .^{2,4,14,19-22}$ Abramson and Sexton ${ }^{4}$ reported a median cost of $\$ 27,083$ (range, $\$ 7,228-\$ 164,392)$. The cost distribution reported in their study represented the median distribution among published studies; therefore, it was chosen as the basis of this study. In our model, we assigned a lognormal distribution to the range reported by Abramson and Sexton. Using the lower and upper limits of the published range as our $5 \%$ and $95 \%$ CI values, we generated a theoretical cost distribution curve with mean and median MRSA infection costs of $\$ 54,153$ and $\$ 34,494$, respectively. See Table 1 for model inputs and calculations.

Model calculations. The daily noncompliant direct contact (NDC) rate was calculated by multiplying daily contacts by (1 - compliance rate). The mean NDC rate was calculated to be 14.50 (95\% CI, 13.26-15.77) per patient-day.

A contaminated encounter (CE) was defined as an NDC in which transmission of MRSA might occur; that is, the first patient contacted by the healthcare worker in the scenario was MRSA-positive and the second patient contacted was MRSA-negative. For simplification, healthcare workers were assumed to be MRSA-negative before contact with the first patient. The expected number of CEs per hospital stay was calculated as the product of the daily NDC rate, the probability that the NDC was a CE, and the mean length of a hospital stay in days. On the basis of these calculations, we estimated that the expected number of CEs per patient-day was $0.64(95 \% \mathrm{CI}, 0.57-0.71)$ and that the expected number of CEs per hospital stay was equal to $0.64 \times 6.26=4.01$. The expected rate of MRSA colonization per CE (MRSA/ $\mathrm{CE}$ ) was calculated by dividing the prevalence of hospitalacquired MRSA by the number of CEs per hospital stay: $0.0143 / 4.01=0.0036($ Table 1$)$.

\section{Simulation}

Simulation design. One million NDCs were simulated. The simulation model determined (1) whether an MRSA infection occurred after an NDC and (2) the cost of the subsequent infection. Total infection costs were then compiled and amortized over all 1 million NDCs to calculate a mean cost per NDC. The simulation was executed for 2 scenarios, a normal risk scenario (the MRSA status of both patients is unknown) and a high-risk scenario (patient 1 has positive MRSA status).

The simulation model used a set of 4 Boolean variables to define a single path for hospital-acquired MRSA infection (see Figure 1, for a detailed simulation flow, and Table 2). Boolean variables included the probability that patient 1 is colonized with MRSA $(P=0.0463)$, the probability that patient 2 is not colonized with MRSA $(P=0.9537)$, the probability of MRSA transmission $(P=0.0036)$, and the probability of MRSA infection $(P=0.29)$. Variables are summarized in Table 3. In the event that a simulated infection occurred, a cost was assigned to the event on the basis of a lognormal distribution (mean, \$54,064; median, \$34,459; 95\% CI, \$7,228-\$164,392).

Normal and high-risk scenarios. In the normal risk scenario, the MRSA status of patient 1 was unknown. Therefore, all 4 Boolean variables were used to determine whether an infection occurred. In contrast, in the high-risk scenario patient 1 was assumed to be colonized or infected with MRSA. In this case, only 3 Boolean variables were included in the simulation (ie, patient 1 MRSA status, $P=1.0$ ) (Figure 1).

Alternative model. Transmission of hospital-acquired MRSA is not caused exclusively by direct patient contact. While the role of the environment in MRSA transmission is not completely understood, studies have successfully isolated MRSA from environmental surfaces in rooms occupied by patients who are colonized with MRSA..$^{23}$ One study demonstrated an increased incidence of MRSA acquisition by patients who stayed in rooms that had previously been occupied by patients who were colonized or infected with MRSA. ${ }^{24}$ Thus, it is likely that some hospital-acquired MRSA infections are caused by contact with contaminated environmental surfaces rather than by direct contact with a healthcare worker. Environmental surfaces can also become contaminated with MRSA during a patient visit by a healthcare worker who is colonized with MRSA. As a result, contamination of the environment can occur during a patient visit that does not involve direct patient contact (ie, during a routine visit, not necessarily during a $\mathrm{CE}$ ). To address this environmental contamination scenario, we included a simulation based on the total number of room visits instead of the number of direct patient contacts only. This model derivation assumed 

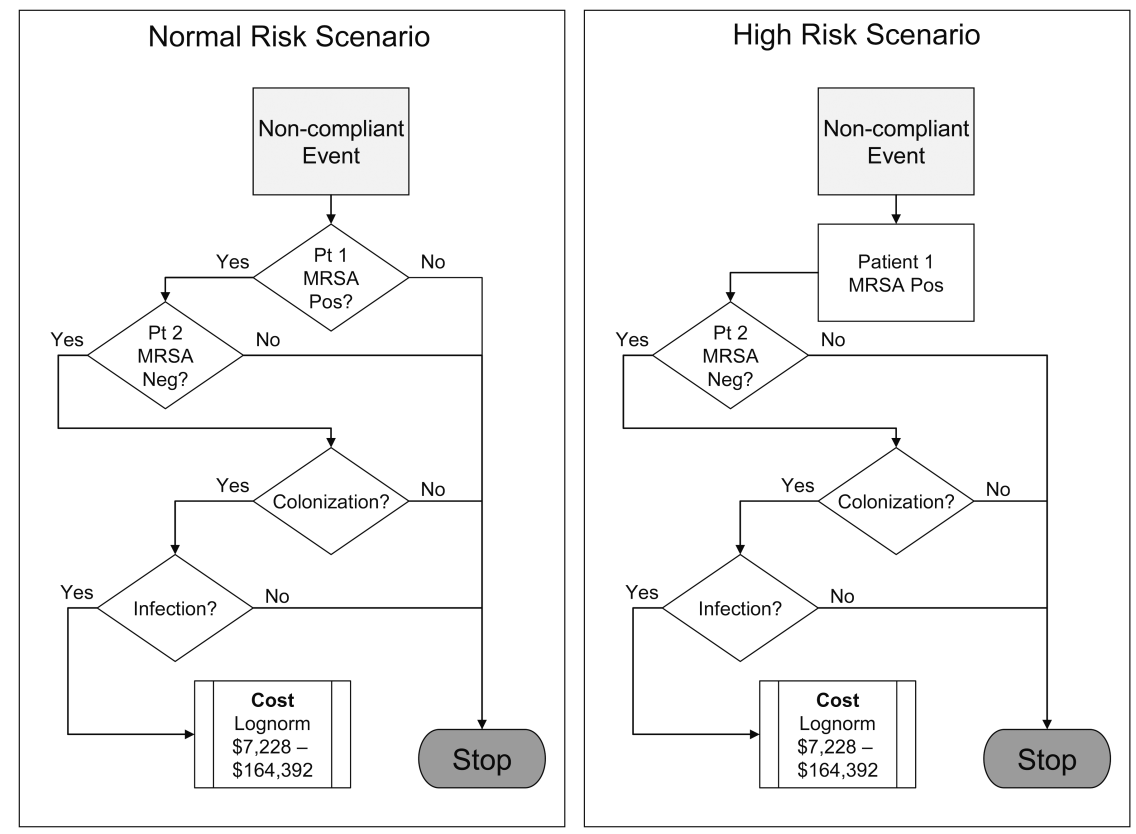

FIGURE 1. Simulation logic for likelihood of transmission of methicillin-resistant Staphylococcus aureus (MRSA) between 2 patients by the hands of healthcare workers. The normal risk scenario involves hand hygiene noncompliance by a healthcare worker between contact with 2 patients of unknown MRSA status. The high-risk scenario involves hand hygiene noncompliance between 2 patient contacts by a healthcare worker during which patient 1 was colonized or infected with MRSA and the MRSA status of patient 2 was unknown. Descriptions and probabilities for both scenarios are shown in Table 2. Pt, patient; Pos, positive; Neg, negative.

that each noncompliant event exhibited an equal probability of MRSA transmission regardless of whether direct patient contact occurred. In this model, we used the Dedrick ${ }^{18}$ estimate for hand hygiene compliance of $45.1 \%$ after a patient room visit (not limited to direct patient contact). We used Duke data for mean daily room visits of 56.38 per patientday (not limited to room visits with direct contact) as the basis for the number of room visits.

Secondary analysis. Simulation results were applied to a hypothetical 200-bed hospital operating at $85 \%$ occupancy with MRSA prevalence and hand hygiene compliance equal to national averages and patient contact rates equal to those of Duke University Medical Center estimates. In this secondary analysis, all patients in the hospital were assumed to have an unknown MRSA status. The expected annual hospital cost attributable to MRSA infection was calculated. Sensitiv- ity analysis was performed on the hand hygiene compliance rate to determine the cost benefit of increasing hand hygiene compliance by $1 \%$.

\section{RES U L T S}

\section{Normal Risk Scenario}

The normal risk scenario is defined as hand hygiene noncompliance by a healthcare worker between contacts with 2 patients of unknown MRSA status. The normal risk simulation resulted in 44,284 CEs over the course of 1 million NDCs (Table 4). Subsequently, 143 episodes of hospital-acquired MRSA colonization occurred, resulting in 42 hospitalacquired MRSA infections. The mean cost per MRSA infection was $\$ 47,092$ (95\% CI, $\$ 26,040-\$ 68,146)$. The median cost per infection was $\$ 22,353$ (interquartile range [IQR],

TA B LE 2. Simulation Logic for Likelihood of Transmission of Methicillin-Resistant Staphylococcus aureus (MRSA) between 2 Patients by the Hands of a Healthcare Worker (HCW)

\begin{tabular}{lllc}
\hline Step & \multicolumn{1}{c}{ Event } & \multicolumn{1}{c}{ Description } & Probability \\
\hline 1 & Noncompliant event & HCW is noncompliant with hand hygiene between 2 consecutive patient encounters & 1.0 \\
2 & Patient 1 MRSA-positive? & First patient encountered is MRSA-positive & 0.0463 or $1.0^{\mathrm{a}}$ \\
3 & Patient 2 MRSA-negative? & Second patient encountered is MRSA-negative & 0.9537 \\
4 & Colonization? & Patient 2 colonized by MRSA as a result of HCW encounter & 0.0036 \\
5 & Infection? & Colonized patient 2 develops infection & 0.29 \\
\hline
\end{tabular}

${ }^{a}$ The normal risk scenario involved patients of unknown MRSA status; community prevalence is $4.63 \%$. The high-risk scenario involved initial patients who were known to be positive for MRSA. 
TAвLE 3. Boolean and Lognormal Variables for Simulation Model

\begin{tabular}{|c|c|c|c|}
\hline Value & $\begin{array}{l}\text { Variable } \\
\text { type }\end{array}$ & $\begin{array}{l}\text { Normal } \\
\text { scenario }\end{array}$ & $\begin{array}{l}\text { High-risk } \\
\text { scenario }\end{array}$ \\
\hline Patient 1 colonized $^{\mathrm{a}}$ & Boolean & 0.0463 & 1.0 \\
\hline Patient 2 uncolonized $^{\mathrm{b}}$ & Boolean & 0.9537 & 0.9537 \\
\hline Colonizations per contaminated encounter ${ }^{c}$ & Boolean & 0.0036 & 0.0036 \\
\hline Infections per colonization ${ }^{\mathrm{d}}$ & Boolean & 0.29 & 0.29 \\
\hline Cost per infection, ${ }^{\mathrm{e}} 95 \% \mathrm{CI}, \$$ & Lognormal & $7,228-164,392$ & $7,228-164,392$ \\
\hline
\end{tabular}

NOTE. CI, confidence interval; MRSA, methicillin-resistant Staphylococcus aureus.

a Probability that patient 1 is colonized with MRSA.

${ }^{b}$ Probability that patient 2 is not colonized with MRSA ( 1 - [patient 1 colonized]).

c Probability of patient 2 colonization given a contaminated encounter.

d Probability of patient 2 infection given patient 2 colonization.

e Cost given patient 2 infection.

$\$ 17,006-\$ 42,996)$. The mean cost per NDC was thus $\$ 1.98$ (95\% CI, \$0.91-\$3.04).

\section{High-Risk Scenario}

The high-risk scenario is defined as hand hygiene noncompliance between 2 patient contacts by a healthcare worker during which patient 1 was colonized or infected with MRSA and the MRSA status of patient 2 was unknown. The highrisk simulation resulted in 953,912 CEs over the course of 1 million NDCs, resulting in 3,340 episodes of hospital-acquired MRSA colonization and 980 episodes of hospital-acquired MRSA infection (Table 4). These infections resulted in a mean cost of $\$ 53,598(95 \%$ CI, $\$ 50,098-\$ 57,097)$ and a median cost of $\$ 35,045$ (IQR, $\$ 18,106-\$ 72,022$ ). The mean cost per NDC was thus $\$ 52.53$ (95\% CI, \$47.73-\$57.32).

\section{Alternative Model}

The alternative model is a simulation based on the total number of room visits (instead of direct patient contacts only). The alternative normal risk simulation resulted in 44,173 CEs over the course of 1 million NDCs, resulting in 83 episodes of hospital-acquired MRSA colonization and 27 episodes of MRSA infection (Table 4). The mean cost of MRSA infection was $\$ 57,442$ (95\% CI, $\$ 23,299-\$ 91,585)$. The median cost of MRSA infection was $\$ 30,458$ (IQR, $\$ 23,291-52,615)$. The mean cost per NDC in the alternative normal risk model was thus $\$ 1.55$ (95\% CI, \$0.47-\$2.63).

\section{Secondary Analysis}

The simulation results were applied to a hypothetical 200bed hospital. A 200 -bed hospital at $85 \%$ occupancy provides care for approximately 62,050 patient-days per year. Each patient-day involves 32.27 direct patient contacts with $55.13 \%$ hand hygiene compliance, resulting in 14.50 NDCs per patient-day, or 899,581 annual NDCs per 200-bed facility. This translates to an estimated 37.8 hospital-acquired MRSA infections annually and an annual cost related to hospital-acquired MRSA infection of $\$ 1,779,283$ (95\% CI, $\$ 1,231,160$ $\$ 2,378,120)$. Increasing hand hygiene compliance by $1 \%$ resulted in a decrease of annual NDCs by 20,046, prevention of 0.84 MRSA infection, and a mean decrease in expected MRSA-related costs of $\$ 39,650$ (95\% CI, $\$ 18,286-\$ 61,014$ ). A 5\% improvement in hand hygiene compliance resulted in a decrease of annual NDCs by 100,232, prevention of 4.21 MRSA infections, and a mean decrease in expected MRSArelated costs of $\$ 198,250$ (95\% CI, $\$ 91,429-\$ 305,072)$.

тAвLE 4. Results of Normal Risk, High-Risk, and Alternative Normal Risk Simulations

\begin{tabular}{lccr}
\hline Result & $\begin{array}{c}\text { Normal risk } \\
\text { scenario }^{\mathrm{a}}\end{array}$ & $\begin{array}{c}\text { High-risk } \\
\text { scenario }^{\mathrm{b}}\end{array}$ & $\begin{array}{c}\text { Alternative } \\
\text { model }^{\mathrm{c}}\end{array}$ \\
\hline Noncompliant events & $1,000,000$ & $1,000,000$ & $1,000,000$ \\
Contaminated encounters & 44,284 & 953,912 & 44,173 \\
MRSA colonizations & 143 & 3340 & 83 \\
MRSA infections & 42 & 980 & 27 \\
Cost of MRSA infection, \$ & & & $57,442(23,299-91,585)$ \\
$\quad$ Mean (95\% CI) & $47,092(26,040-68,146)$ & $53,598(50,098-57,097)$ & $30,458(23,291-52,615)$ \\
$\quad$ Median (IQR) & $22,353(17,006-42,996)$ & $35,045(18,106-72,022)$ & $1.55(0.47-2.63)$ \\
Cost per noncompliant event, \$ (95\% CI) & $1.98(0.91-3.04)$ & $52.53(47.73-57.32)$ &
\end{tabular}

Note. CI, confidence interval; IQR, interquartile range; MRSA, methicillin-resistant Staphylococcus aureus.

${ }^{a}$ Hand hygiene noncompliance by a healthcare worker between contact with 2 patients of unknown MRSA colonization status.

${ }^{\mathrm{b}}$ Hand hygiene noncompliance between contact with 2 patients by a healthcare worker during which patient 1 was colonized or infected with MRSA and the MRSA status of patient 2 was unknown.

c Simulation based on total room visits instead of on direct patient contacts only. 


\section{I S C USS I O N}

Infections are spread to patients in the hospital primarily by means of the hands of healthcare workers. Typically, this occurs when healthcare workers neglect to perform hand hygiene before patient contact. This study quantified the cost associated with a single episode of hand hygiene noncompliance. Costs ranged from approximately $\$ 2$ (when a patient's MRSA colonization or infection status was unknown) to more than $\$ 50$ per episode (when healthcare workers did not wash their hands after contact with a patient who was an MRSA carrier). On the basis of these estimates, improved hand hygiene compliance among healthcare workers in a 200bed hospital by as little as $1 \%$ would prevent approximately 1 episode of infection due to MRSA and would result in MRSA prevention-associated cost savings of almost $\$ 40,000$ per year. If the same hospital improved hand hygiene compliance by $5 \%$, approximately 4 MRSA infections would be prevented and the cost savings would approach $\$ 200,000$.

The findings from this study represent a departure from the conventional method of analyzing costs associated with hospital-acquired infection. Historically, costs have been estimated as a function of number and types of infection., ${ }^{2,40-22}$ Unfortunately, since the transmission of pathogens in the hospital occurs silently, it is impossible to attribute an incident of hospital transmission of a pathogen or the resulting hospitalacquired infection to the behaviors of an individual healthcare worker. Because of this inability to attribute causality to a healthcare worker's actions, it is difficult to make healthcare workers accountable for the occurrence of hospital-acquired infections. Costs presented on a "per infection" basis seem abstract to many clinicians and often are ineffective in generating accountability for behavior and improving compliance among healthcare workers. Conversely, presenting cost as a function of compliance with process (such as hand hygiene) is more relevant and tangible to healthcare workers and, we believe, can be used to elicit greater accountability for and ownership of suboptimal hand hygiene practices.

This study also presented an additional model that accounted for the possibility that a healthcare worker could transfer MRSA from 1 patient to a second patient during a room visit, even if the healthcare worker had contact only with the room environment but no direct contact with the second patient. In this alternative simulation, the cost associated with each episode of hand hygiene noncompliance was $\$ 1.55$.

Several assumptions were made to make the model practical and clinically useful. First, we assumed steady state MRSA prevalence. In reality, MRSA prevalence is likely fluid and has been increasing over time. ${ }^{25}$ We believe, however, that in a short-run experiment the assumption of stable prevalence is reasonable. While short-term fluctuations in MRSA prevalence have not been examined, we believe these fluctuations to be the result of infection outbreaks resulting in increased, rather than decreased, prevalence. Given the like- lihood of MRSA prevalence to increase over time and as a result of nonzero probability of the occurrence of an MRSA outbreak, it is likely that the study results represent an underestimate of the actual cost of hand hygiene noncompliance. Second, we assumed that all MRSA transmission in the hospital resulted from hand hygiene noncompliance. While data show that hand hygiene noncompliance is the leading cause of hospital-acquired MRSA infection, ${ }^{7}$ there are other causes of transmission as well, such as contaminated shared equipment. To the extent that other causes account for hospital-acquired MRSA infection, our results may represent an overestimate of NDC cost. In addition, the model assumed rates of hand hygiene compliance among healthcare workers that were based on published US data. ${ }^{26}$ If higher rates of compliance had been included in the model, the resulting number of MRSA infections and MRSA-associated costs would have been smaller. The model also did not account for the proportion of healthcare workers who might be chronic carriers of MRSA (4.6\% of healthcare workers, according to 1 study).$^{27}$ Finally, some might consider the mean cost per MRSA infection (approximately \$50,000) used in the model to be too high, although we used mean and median values from published literature. In addition, some reports have noted that after patients become colonized with MRSA during hospitalization almost $50 \%$ of MRSA infections occurred after discharge, ${ }^{28}$ and some of these postdischarge infections would not have been accounted for in this model. Thus, MRSA infection-related costs in this model might have been underestimated. Furthermore, even if the cost used in modeling had been as low as one-half the mean value used (approximately $\$ 25,000$ per MRSA infection), the annual MRSA infection-related expenses attributable to hand hygiene noncompliance accrued by a 200-bed hospital would still have been substantial (approximately $\$ 500,000$ ).

Each NDC represented a single episode of hand hygiene noncompliance. Ergo, we assumed that each iteration involved compliant hand hygiene prior to contact with the first patient. We did not account for the possibility that healthcare workers who are habitually noncompliant may have a much higher incidence and burden of contamination or colonization with MRSA. While it is likely that a disproportionate number of transmissions are caused by such a group of habitually noncompliant individuals, the results are averaged over the entire population of healthcare workers. Therefore, we assume that all healthcare workers exhibit the same compliance rate. Again, we believe that this simplification causes our model to underestimate actual costs, because habitually noncompliant workers will display a higher transmission rate resulting from multiple consecutive NDCs. In addition, during the study period it was the policy at Duke University Medical Center to use contact precautions (gowns and gloves for all healthcare workers entering the room) for patients known to be infected or colonized with MRSA for the duration of the hospitalization. The association between the use of contact precautions and the number of direct contacts was 
not studied at Duke during the study period and thus was not incorporated into the model.

Costs associated with hand hygiene noncompliance were limited exclusively to nosocomial MRSA transmission and infection. Many other pathogens are also spread to patients on the hands of healthcare workers as a result of noncompliance with hand hygiene. In fact, in some reports MRSA accounts for fewer than $8 \%$ of all hospital-acquired infections. ${ }^{29}$ Because our model focused on costs associated only with MRSA transmission, it substantially underestimated the costs associated with hand hygiene noncompliance. To form a more complete and accurate estimate of the costs associated with hand hygiene noncompliance, additional analyses should be conducted that focus on costs associated with hospital transmission of other pathogens in addition to MRSA.

Poor practices among healthcare workers lead to patient harm. Unfortunately, these poor practices occur frequently in the hospital. Noncompliance with hand hygiene places patients at unnecessary risk for colonization with and subsequent infection by multidrug-resistant pathogens, such as MRSA. Despite the well-publicized fact that MRSA leads to poor outcomes and increased cost for patients, most hand hygiene campaigns fail to lead to sustained improvements in hand hygiene compliance. This study provides a relatively conservative (yet still alarming) estimate of the financial impact of a single incident of hand hygiene noncompliance and also provides an estimate of the aggregate costs imparted by noncompliance with hand hygiene for a typical US hospital. The results from this study can be used to attribute cost to and improve accountability for suboptimal healthcare worker behaviors. In addition, these results provide cost estimates that can be used to model the cost-effectiveness of hand hygiene interventions and may give hospitals and organizations the incentive to invest in novel and effective methods and technologies for improving the hand hygiene culture, habits, and compliance of healthcare workers.

\section{ACKNOWLEDGMENTS}

Financial support. K.S.K. was supported by grant K23 AG23621-01A1 from the National Institute of Aging and by the John A. Hartford Foundation. This study was sponsored exclusively by the Department of Infectious Disease at Duke University Medical Center.

Potential conflicts of interest. K.S.K. has served as a consultant for Schering-Plough and Ortho McNeil and is a member of the speakers' bureaus for Cubist, Pfizer, Merck, Schering-Plough, and Wyeth Ayerst. D.J.A. has received research grants (unrelated to the present project) from Merck and from Pfizer. All other authors report no conflicts of interest relevant to this article.

Address reprint requests to Keith S. Kaye, MD, MPH, 3990 John R, Detroit, MI 27710 (kkaye@dmc.org).

\section{REFERENCES}

$\rightarrow$ 1. Klevens RM, Edwards JR, Horan T, et al. Estimating health care-associated infections and deaths in U.S. hospitals, 2002. Public Health Rep 2007;122(2):160-166.

$\rightarrow$ 2. Kopp BJ, Nix DE, Armstrong EP. Clinical and economic analysis of meth- icillin-susceptible and -resistant Staphylococcus aureus infections. Ann Pharmacother 2004;38(9):1377-1382.

$\rightarrow$ 3. Smith RL II, Sawyer RG, Pruett TL. Hospital-acquired infections in the surgical intensive care: epidemiology and prevention. Zentralbl Chir 2003; 128(12):1047-1061.

$\rightarrow$ 4. Abramson MA, Sexton DJ. Nosocomial methicillin-resistant and methicillin-susceptible Staphylococcus aureus primary bacteremia: at what costs? Infect Control Hosp Epidemiol 1999;20(6):408-411.

$\rightarrow$ 5. Aragon D, Sole ML, Brown S. Outcomes of an infection prevention project focusing on hand hygiene and isolation practices. AACN Clin Issues 2005;16(2):121-132.

$\rightarrow$ 6. Pittet D, Hugonnet S, Harbarth S, et al; Infection Control Programme. Effectiveness of a hospital-wide programme to improve compliance with hand hygiene. Lancet 2000;356(9238):1307-1312.

$\rightarrow$ 7. Larson EL, et al. An organizational climate intervention associated with increased handwashing and decreased nosocomial infections. Behav Med 2000;26(1):14-22.

$\rightarrow$ 8. Boyce JM, Pittet D. Guideline for hand hygiene in health-care settings: recommendations of the Healthcare Infection Control Practices Advisory Committee and the HIPAC/SHEA/APIC/IDSA Hand Hygiene Task Force. Am J Infect Control 2002;30(8):S1-S46.

9. The Joint Commission. 2008 Patient safety goals. The Joint Commission Web site. http://www.jcrinc.com/fpdf/pubs/pdfs/JCReqs/JCP-07-07-S1 .pdf. Published 2007. Accessed May 28, 2008.

10. World Health Organization. WHO guidelines on hand hygiene in health care (advanced draft): a summary. World Health Organization Web site. http://www.who.int/patientsafety/events/05/HH_en.pdf. Published 2005. Accessed May 28, 2008.

11. Wullenweber M, Martiny H, Lenz W, et al. Nosocomial infective agents in a surgical intensive care unit. III. Spreading of Staphylococcus aureus with regard to phage typing analysis [in German]. Zentralbl Bakteriol Mikrobiol Hyg [B] 1981;174(6):498-508.

12. Centers for Disease Control and Prevention. Guidelines for hand hygiene in healthcare settings-2002. Centers for Disease Control and Prevention Web site. http://cdc.gov/handhygiene/. Accessed January 30, 2009.

13. Pittet D. Improving adherence to hand hygiene practice: a multidisciplinary approach. Emerg Infect Dis 2001;7(2):234-240.

14. Mauldin PD, Salgado CD, Durkalski VL, et al. Nosocomial infections due to methicillin-resistant Staphylococcus aureus and vancomycin-resistant Enterococcus: relationships with antibiotic use and cost drivers. Ann Pharmacother 2008;42(3):317-326.

15. Anderson DJ, Kirkland KB, Kaye KS, et al. Underresourced hospital infection control and prevention programs: penny wise, pound foolish? Infect Control Hosp Epidemiol 2007;28(7):767-773.

$\rightarrow$ 16. Engemann JJ, Carmeli Y, Cosgrove SE, et al. Adverse clinical and economic outcomes attributable to methicillin resistance among patients with Staphylococcus aureus surgical site infection. Clin Infect Dis 2003;36(5):592-598.

$\rightarrow$ 17. Jarvis WR, Schlosser J, Chinn RY, et al. National prevalence of methicillin-resistant Staphylococcus aureus in inpatients at US health care facilities, 2006. Am J Infect Control 2007;35(10):631-637.

18. Dedrick RE, Sinkowitz-Cochran RL, Cunningham C, et al. Hand hygiene practices after brief encounters with patients: an important opportunity for prevention. Infect Control Hosp Epidemiol 2007;28(3):341-345.

19. Cosgrove SE, Qi Y, Kaye KS, et al. The impact of methicillin resistance in Staphylococcus aureus bacteremia on patient outcomes: mortality, length of stay, and hospital charges. Infect Control Hosp Epidemiol 2005;26(2):166174 .

20. Chaix C, Durand-Zaleski I, Alberti C, et al. Control of endemic methicillin-resistant Staphylococcus aureus: a cost-benefit analysis in an intensive care unit. JAMA 1999;282(18):1745-1751.

21. Lodise TP, McKinnon PS. Clinical and economic impact of methicillin resistance in patients with Staphylococcus aureus bacteremia. Diagn Microbiol Infect Dis 2005;52(2):113-122.

22. Shorr AF, Tabak YP, Gupta V, Johannes RS, Liu LZ, Kollef MH. Morbidity and cost burden of methicillin-resistant Staphylococcus aureus in early onset ventilator-associated pneumonia. Crit Care 2006;10(3):article 
R97. http://ccforum.com/content/10/3/R97. Published June 29, 2006. $\rightarrow$ 27. Albrich WC, Harbarth S. Health-care workers: source, vector, or victim Accessed January 30, 2009. of MRSA? Lancet Infect Dis 2008;8(5):289-301.

$\rightarrow$ 23. Fitzpatrick F, Murphy OM, Brady A, et al. A purpose built MRSA cohort $\rightarrow 28$. Huang SS, Platt R. Risk of methicillin-resistant Staphylococcus aureus unit. J Hosp Infect 2000;46(4):271-279.

$\rightarrow$ 24. Huang SS, Datta R, Platt R. Risk of acquiring antibiotic-resistant bacteria from prior room occupants. Arch Intern Med 2006;166(18):1945-1951.

$\rightarrow$ 25. Kuehnert MJ, Doyle TJ, Hill HA, et al. Prevalence of Staphylococcus aureus nasal colonization in the United States, 2001-2002. J Infect Dis 2006;193(2): $172-179$.

$\rightarrow$ 26. Larson E. Skin hygiene and infection prevention: more of the same or different approaches? Clin Infect Dis 1999;29(5):1287-1294. infection after previous infection or colonization. Clin Infect Dis 2003;36(3): 281-285.

29. Hidron AI, Edwards JR, Patel J, et al. NHSN annual update: antimicrobial-resistant pathogens associated with healthcare-associated infections: annual summary of data reported to the National Healthcare Safety Network at the Centers for Disease Control and Prevention, 2006-2007. Infect Control Hosp Epidemiol 2008;29(11):996-1011. 